\title{
Hochspezialisierte Viszeralchirurgie: Zentralisiert oder Carte blanche?
}

\section{Martin Fey}

Prof. em. Dr. med., Präsident des Fachorgans der IVHSM, GDK, Senior Consultant, Universitätsklinik für medizinische Onkologie, Inselspital und Universität Bern

Mitte Dezember 2017 startete die breit gestreute Anhörung des Zuteilungsberichts zum Thema hochspezialisierte Viszeralchirurgie (HSM VISZ) mit den Vorschlägen zur erneuten Zuteilung von HSM-Leistungsaufträgen an selektionierte Schweizer Kliniken. Da dieses Geschäft auf breites Interesse, aber auch auf bisweilen vehemente Kritik stossen mag, ist es opportun, die Steuerung der hochspezialisierten Medizin (HSM) in der Schweiz mit Argumenten zugunsten einer Zentralisierung der HSM VISZ darzustellen.

\section{IVHSM: Grundlagen}

Mit der Interkantonalen Vereinbarung über die hochspezialisierte Medizin (IVHSM) aller Kantone wurde im Jahre 2008 «im Interesse einer bedarfsgerechten, qualitativ hochstehenden und wirtschaftlich erbrachten medizinischen Versorgung die Konzentration der hochspezialisierten Medizin (HSM)» geregelt. Ziel der IVHSM war und ist es, medizinische Bereiche und Leistungen, die durch ihre Seltenheit, ihr hohes Innovationspotential, ihren hohen personellen oder technischen Aufwand oder durch komplexe Behandlungsverfahren gekennzeichnet sind, zu konzentrieren und schrittweise nur wenigen universitären oder multidisziplinären Spitalzentren in der Schweiz zuzuteilen. Die IVHSM stützt sich auf Art. 39 des Krankenversicherungsgesetzes (KVG), der im Absatz 1 festhält, dass die stationäre medizinische Betreuung zu regeln sei-daraus kann abgeleitet werden, dass ambulante Medizin

\section{Résumé}

La chirurgie de l'œsophage, du pancréas, du rectum profond et la chirurgie hépatique et bariatrique complexe sont rattachées à la médecine hautement spécialisée (MHS). Un rapport comprenant des propositions d'attribution de nouveaux mandats de prestations a récemment été rédigé par l'Organe scientifique MHS et approuvé pour audition par I'Organe de décision MHS. L'audition a lieu de la mi-décembre 2017 à la fin janvier 2018. Cet article expose les réserves et les arguments contre la réglementation MHS de la chirurgie viscérale hautement spécialisée en Suisse. Les partisans MHS mettent en avant l'assurance qualité clinique, de bonnes conditions de formation approfondie en chirurgie viscérale ainsi qu'un contexte favorable à la recherche clinique. den IVHSM-Vorgaben nicht unterstehen dürfte, auch wenn dies nicht expressis verbis festgehalten ist. Die HSM-Gremien sind das Beschlussorgan (BO) und das Fachorgan (FO). Im HSM-BO nehmen die Gesundheitsdirektoren/-innen (GD) von fünf Universitätskantonen, ferner die GD von mindestens zwei Kantonen mit grossen Zentrumsspitälern und von drei weiteren Kantonen Einsitz. Das HSM-FO setzt sich aus medizinischen Experten/-innen aus dem In- und Ausland zusammen. Die Begleitgruppen rekrutieren sich aus Mitgliedern der entsprechenden Fachgesellschaften. Auf die personelle Zusammensetzung einer Begleitgruppe nehmen weder das FO noch das BO Einfluss. Gestützt auf Anträge der fachlich zuständigen Begleitgruppe identifiziert das FO Indikationen und Eingriffe (markiert durch CHOP- und ICD-Codes) für die Zuordnung zur HSM; der Zuordnungsbeschluss obliegt dem BO. In einem zweiten Schritt legt das FO, beraten durch die Begleitgruppe, die Voraussetzungen und Kriterien fest, welche die Bewerber für die Zuteilung eines HSM-Leistungsauftrags zwingend erfüllen müssen. Gestützt auf die Anträge des FO beschliesst das BO schliesslich die HSM-Zuteilung an Spitäler, die die vorgegebenen Kriterien erfüllen. Selbstverständlich sind breit gestreute Anhörungen in diversen Etappen Pflicht.

Anlässlich der Gründung der IVHSM wurde eine «beispielhafte» Liste von medizinischen HSM-Bereichen erstellt, mit dem Hinweis, sie sei keinesfalls abschliessend. Viele dieser Themen wurden bisher erfolgreich angegangen, so die Transplantationsmedizin, die Behandlung schwerer Verbrennungen oder die pädiatrische Onkologie. Andere Themen aus der «Gründerliste» wurden bewusst nicht aufgenommen, z.B. die PET-Diagnostik (ein ambulanter diagnostischer Service) oder die Behandlung der Augenmelanome (die ohne Zutun der HSM mehrheitlich im CHUV in Lausanne zentriert ist). Noch andere Gebiete, wie die sehr seltenen Operationen zur Geschlechtsumwandlung, boten bisher keinen Handlungsbedarf, und "die Molekulargenetik», wiewohl auf der Liste, lässt sich nicht mit CHOP- und ICD-Codes als HSM-Fach eingrenzen. 
Tabelle 1: HSM-Zuordnungen in der Viszeralchirurgie gemäss Beschluss des BO vom 21.1.2016.

\begin{tabular}{|c|c|c|c|}
\hline Bereich laut BO-Beschluss & Relevante Diagnosen & Eingriffe (zusammengefasst) & Kommentare \\
\hline Tiefe Rektum-Resektion & $\begin{array}{l}\text { Tiefliegende } \\
\text { (praktisch immer maligne) } \\
\text { Rektum- und Analtumoren }\end{array}$ & $\begin{array}{l}\text { Tiefe anteriore Rektumresektion, } \\
\text { abdomino-perineale Rektumresektion, } \\
\text { totale Prokto-Kolektomie }\end{array}$ & $\begin{array}{l}\text { Primärtumor 0-12 cm ab ano, in Anlehnung } \\
\text { an Vorgaben der Deutschen Krebsgesell- } \\
\text { schaft }\end{array}$ \\
\hline Pankreasresektion & $\begin{array}{l}\text { Pankreaskarzinom, } \\
\text { chronische Pankreatitis }\end{array}$ & $\begin{array}{l}\text { Totale oder partielle Pankreatektomie, } \\
\text { Exzision der Ampulla Vateri }\end{array}$ & $\begin{array}{l}\text { Drainage-Eingriffe sowie palliative Eingriffe } \\
\text { unterstehen nicht HSM VISZ }\end{array}$ \\
\hline Oesophagusresektion & $\begin{array}{l}\text { Oesophaguskarzinom, } \\
\text { und andere Pathologien }\end{array}$ & $\begin{array}{l}\text { Partielle oder totale Oesophagektomie, } \\
\text { intrathorakale Anastomosen }\end{array}$ & $\begin{array}{l}\text { Totale Gastrektomie nicht der HSM VISZ } \\
\text { zugeordnet }\end{array}$ \\
\hline Leberresektion & $\begin{array}{l}\text { Lebertumoren } \\
\text { (maligne und benigne) }\end{array}$ & $\begin{array}{l}\text { Anatomische Leberresektion wie Hemi- } \\
\text { hepatektomien oder Bisegmentektomien, } \\
\text { Leberexzisionen von mehreren Herden, } \\
\text { laparoskopische Exzisionen u.a.m. }\end{array}$ & $\begin{array}{l}\text { Kleinere Resektionen von 1-2 Herden oder } \\
\text { Leberzysten-Resektionen unterstehen nicht } \\
\text { der HSM VISZ }\end{array}$ \\
\hline $\begin{array}{l}\text { Komplexe bariatrische } \\
\text { Chirurgie }\end{array}$ & $\begin{array}{l}\text { Exzessive Adipositas mit } \\
\text { zusätzlichen Risikofaktoren }\end{array}$ & $\begin{array}{l}\text { Malabsorptive Eingriffe (bilio-pankreatische } \\
\text { Diversionen), Magenbandrepositionen, } \\
\text { bariatrische Revisionseingriffe }\end{array}$ & $\begin{array}{l}\text { Nur komplexe Eingriffe gemäss SMOB-Defi- } \\
\text { nitionen, ferner vor allem Revisionseingriffe, } \\
\text { Patienten mit Risikofaktoren (besonders } \\
\text { hoher BMI, ASA-Score) }\end{array}$ \\
\hline
\end{tabular}

\section{Der Knackpunkt der hochspezialisierten Viszeralchirurgie}

Fünf Themen aus der Viszeralchirurgie (HSM VISZ) wurden im Jahre 2013 der HSM zugeordnet (Tab. 1), und gleichzeitig wurden die Spitäler zur Bewerbung eingeladen. Das Prozedere führte zu zahlreichen Protesten und Rechtsfällen, die die weiteren Arbeiten zunächst behinderten, teilweise aber auch Klärung prozeduraler Abläufe mit sich brachten. Das Bundesverwaltungsgericht hatte bereits im HSM-Thema der komplexen Behandlung von Hirnschlägen ein wegweisendes Urteil gefällt, wonach eine erfolgte Zuordnung gerichtlich nicht mehr angefochten werden kann. Eine Folge der Wirren um die Viszeralchirurgie war das heutige zweistufige Verfahren. Demnach wird in einer ersten Stufe die Zuordnung eines Gebiets zur HSM verbindlich festgelegt. In einer zweiten Stufe erfolgt eine Einladung an die Spitäler zur Bewerbung um Zuteilung des HSMLeistungsauftrags. Ihre Bewerbungen werden aufgrund vorgegebener Kriterien vom FO und von der Begleitgruppe überprüft. Das BO legt schliesslich die Zuteilungen fest.

Die fünf der HSM zugeordneten Gebiete aus der Viszeralchirurgie (Tab. 1) erfüllen alle HSM-Kriterien. Trotzdem wirft gerade HSM VISZ weiterhin hohe Wellen, und das Thema ist für einige Kritiker der Anlass, die IVHSM zu verwässern oder ganz zu beerdigen. Die Gründe, gegen HSM VISZ Sturm zu laufen wie weiland die Eidgenossen gegen das Haus Habsburg, sind wohl in folgenden Aspekten zu orten. Im Gegensatz beispielsweise zur allogenen Stammzelltransplantation, die von jeher nur an drei Unikliniken angeboten wird (HSM-bestätigt), führen in der Schweiz viele Spitäler viszeralchirurgische Kliniken. Viele davon betreuen seit langem Patienten mit Diagnosen und Eingriffen, die nun der HSM VISZ zugeordnet wurden. Wie das aktuelle Bewerbungsverfahren zeigt, weisen die Hälfte der fast 30 Kliniken, die sich beispielsweise um eine Zuteilung für Pankreaskarzinom-Chirurgie beworben haben, Fallzahlen von weniger als zehn Patienten pro Jahr auf; ein Drittel der Kliniken führen derartige Eingriffe bloss zwei- bis dreimal jährlich durch. Diese Spitäler halten trotz tiefer Fallzahl offenbar am Anspruch fest, diese Eingriffe selten einmal vornehmen zu dürfen. Ihre Chefchirurgen empfinden sie wohl als spannende Herausforderung, und die Spitaldirektoren stufen sie als prestigeträchtiges lokales Angebot ein, ferner als Anreiz für die Rekrutierung qualifizierter Klinikmitarbeiter.

\section{Minimale Fallzahlen als Qualitätsgarant in der Viszeralchirurgie}

Im Jahre 2002 wiesen Birkmeyer et al. in einer wegweisenden Arbeit nach, dass Spitäler mit hohen Patientenzahlen (high-volume hospitals) bei diversen kardiologischen und onkologischen Eingriffen deutlich bessere Resultate erzielten als Krankenhäuser mit Patientenzahlen unterhalb einer kritischen Grenze [1]. Die Daten dieser Pionierarbeit wurden in zahlreichen Studien bestätigt, so auch für die HSM-VISZ-Eingriffe. Trotzdem blieben diese Erkenntnisse in der Schweizer Spitallandschaft praktisch folgenlos, wie wenn sie für den «Sonderfall Schweiz» keine Geltung hätten. Dabei fehlte es nicht an Studien, die auch bei Schweizer Patienten belegen, dass repetitive chirurgische Praxis bessere Resultate erzielt. Schweizer Kohortenstudien zum Thema kolorektaler Karzinome zeigten auf, dass ein Spital mit hohen Patienten- und Eingriffszahlen (>25 jährlich pro Spital) ein deutlich besseres rezidivfreies und Gesamtüberleben erzielt [2]. Kürzlich wiesen nun Güller und Kollegen mit einer flächendeckenden Analyse aller 
Schweizer Spitäler nach, dass die postoperative Frühmortalität nach Oesophagus-, Pankreas- und Rektumkarzinom-Chirurgie in Kliniken, die diese Eingriffe nur selten durchführen, deutlich höher ist als in Zentren mit höherem Patientenaufkommen [3]. Man sollte meinen, dass die Botschaft nun auch in der Schweizer Chirurgie- und Spitalszene respektiert werden müsste, wiewohl nicht allen Akteuren genehm. Die im Bereich HSM VISZ festgesetzten minimalen Fallzahlen von 12 Eingriffen pro Jahr entsprechen ohnehin einem Kompromiss, da sie mehr der politischen Opportunität gehorchend denn der wissenschaftlichen Datenlage folgend so tief angesetzt wurden. Auch wenn offenbar der Verzicht auf HSM-VISZ-Eingriffe für viele Spitäler schmerzhaft ist, so ist er ein wichtiger und richtiger Weg zur Qualitätsverbesserung, wenn Patienten mit Oesophagus-, Pankreas- und Rektumkarzinomen in kleineren Schweizer Spitälern nicht weiterhin ein zu hohes Sterberisiko gewärtigen sollen.

Gelegentlich wird argumentiert, die Auflage, minimale Fallzahlen pro Jahr zu erfüllen, führe zu unkritischer Indikationsstellung. So mag denn eine Klinik, der gegen Ende eines Kalenderjahres zur Erfüllung der magischen HSM-Schwelle von 12 Patienten mit Rektum- oder Pankreaskarzinom noch je zwei Fälle fehlen, versuchen, diese Patienten durch "grosszügige» Indikationsstellung doch noch zeitgerecht auf den OP-Tisch zu kriegen. Die Lösung dieses Problems ist jedoch nicht, minimale Fallzahlen abzuschaffen, sondern sie in Zukunft zu erhöhen. Grosse Zentren mit ohnehin hohem case load erreichen hohe minimale Fallzahlen auch mit kritischer OP-Indikation - damit wird gute evidenzbasierte Chirurgie betrieben und optimale Qualität erreicht, was just Ziel und Zweck von HSM VISZ ist.

\section{Der «Sonderfall» Rektumkarzinom}

Einzelne HSM-Kritiker zweifeln, ob die Zuordnung der tiefen anterioren Rektumresektion zur HSM wirklich gerechtfertigt sei. Die zitierte Studie von Güller weist auch beim Rektumkarzinom eine erhöhte Frühmortalität in Kliniken mit tiefem case load auf [3]. Ferner korrelieren die onkologischen Langzeitresultate beim tiefen Rektumkarzinom mit dem case load - ein weiteres stichhaltiges Argument, dass nur multidisziplinäre Teams mit regelmässiger Praxis diese schwierigen Therapien durchführen sollten [4]. Es wurde nun der Vorschlag eingebracht, nur die Therapie von Rektumkarzinomen im Bereich von 0-6 $\mathrm{cm} a b$ ano (statt 0-12 cm) der HSM zuzuordnen. Rektumkarzinome, die somit einer Palpation mit dem Zeigefinger nicht zugänglich sind, wären aus der HSM-Zuordnung zu streichen. Die totale mesorektale Rektumresektion wegen
Karzinom im mittleren Rektum ist jedoch nicht wesentlich einfacher - vielmehr hängen die onkologischen Resultate im ganzen Rektum von einer qualitativ hochstehenden und regen chirurgischen Praxis ab. Motiv und Ziel dieses Vorschlags dürfte ja sichtlich nicht die Sorge um Qualität sein, sondern die Möglichkeit für kleinere Kliniken, aus Image-Gründen mindestens einen Teil der Rektumkarzinom-Chirurgie behalten zu dürfen.

\section{HSM Viszeralchirurgie und chirurgische Grundversorgung an den Spitälern}

Oft und gerne wird behauptet, ein Spital ohne Leistungsauftrag in HSM VISZ könne die chirurgische Grundversorgung im Einzugsgebiet nicht mehr sicherstellen. Gute Chirurgie-Chefärzte, denen die Möglichkeit verwehrt wäre, ihre wenigen prestigeträchtigen HSM-VISZ-Eingriffe (wie beispielsweise eine Pankreatektomie oder eine tiefe Rektumresektion) zu praktizieren, würden dem zurückgestutzten Spital den Rücken kehren. Ihre Nachfolge sei wohl kaum mehr zu gewährleisten.

Das Programm «HSM Viszeralchirurgie» (Tab. 1) regelt gerade einmal 1\% aller viszeralchirurgischen Eingriffe in unseren Spitälern. Es fällt schwer, zu verstehen, weshalb dieser Verzicht die qualitativ hochstehende Praxis der restlichen 99\% aller Eingriffe gefährden und die Chefchirurgen völlig demotivieren sollte. Unter diese 99\% von "Non HSM VISZ» fallen zahlreiche Eingriffe, denen es an "chirurgischem Prestige» keinesfalls mangelt (wenn denn dieses Kriterium überhaupt von Relevanz wäre), beispielsweise Hemikolektomien oder die Leberchirurgie, die nicht unter die wenigen besonders heiklen HSM-Leberresektionen fällt.

\section{Die Weiterbildung zum Chirurgen - und zum Chirurgen mit Schwerpunkt Viszeralchirurgie}

Viele Kliniken in der Schweiz bekunden Schwierigkeiten, Assistenzärzte zu rekrutieren. Dieses Problem ist jedoch nicht HSM-bedingt, sondern helvetisch hausgemacht. Der Bedarf an jungen Ärzten in unseren Spitälern steigt im Zuge gesellschaftlicher Trends wegen mehr Teilzeitstellen und wegen der Arbeitszeitbeschränkung. Die Ärzteproduktion unserer $\mathrm{Fa}$ kultäten hält jedoch mit den Erfordernissen des Arbeitsmarkts nicht Schritt. Die Billiglösung, fehlenden Nachwuchs aus dem Ausland zu rekrutieren, strandet zunehmend; der Ansturm auf Schweizer Assistenzarztstellen von Bewerbern aus Deutschland beispielsweise hat deutlich abgenommen. Aus diesem 
Problemkreis heraus HSM VISZ abzulehnen ist weder logisch noch zielführend.

Man hört, in Kliniken ohne HSM-VISZ-Zuteilung sei die Weiterbildung in Chirurgie gefährdet. Die fünf HSMEingriffe fallen jedoch nicht in den Operationskatalog, der für den Facharzt in Chirurgie erfüllt werden muss. Für die chirurgische Grundweiterbildung ist die HSMZuteilung somit irrelevant.

Für die Weiterbildung im Schwerpunkt Viszeralchirurgie bestehen drei Klassen von anerkannten Weiterbildungsstätten (V1-3). Obwohl erfolgreiche Bewerber um HSM-VISZ-Leistungsaufträge die Anerkennung als Weiterbildungsstätte nachweisen müssen (gelegentlich ein Stolperstein für Privatkliniken), sind alle drei Kategorien anerkannt, zum Vorteil kleinerer Kliniken. Die Zentralisierung von viszeralen HSM-Eingriffen an grösseren Kliniken ist eine wichtige Voraussetzung für eine effiziente Weiterbildung im Schwerpunkt Viszeralchirurgie, weil Chirurgie repetitiv erlernt werden muss (welcher Berufspianist würde denn ohne tägliches fleissiges Üben die Chopin-Etüden beherrschen?). Aus diesen Überlegungen darf man skalpellscharf schliessen, dass die HSM-Zentralisierung der fünf Bereiche der Viszeralchirurgie auf wenige grosse Kliniken eine wichtige Voraussetzung für eine effiziente Weiterbildung im Schwerpunkt Viszeralchirurgie ist.

\section{Die Förderung klinischer Studien an Schweizer Spitälern}

HSM will klinische Forschung fördern, die in der Schweiz einen international geringeren Leistungsausweis hat als die experimentelle Spitzenforschung. Die Auflagen an die erfolgreiche Durchführung klinischer Studien sind wegen des Humanforschungsgesetzes
(HFG) deutlich verschärft worden, und viele kleinere und mittelgrosse Spitäler können oder wollen sie nicht (mehr) erfüllen. Folglich bietet die Zentralisierung von HSM VISZ auf wenige Grossanbieter bessere Voraussetzungen für klinische Forschung.

\section{Fazit}

Regionale Spitäler erfüllen weiterhin eine wichtige Funktion in der Versorgungslandschaft der Schweiz. Ihre Stellung mit einem kleinen Kontingent an hochspezialisierten medizinischen Behandlungen in der Viszeralchirurgie erhärten zu wollen ist jedoch nicht zielführend. Im Interesse der betroffenen Patientinnen und Patienten sollte die vorgeschlagene Zuteilung der fünf HSM-VISZ-Bereiche nun umgesetzt werden.

Der Inhalt des Manuskripts gibt meine persönliche Meinung wieder.

Ich danke dem Präsidenten des HSM-Beschlussorgans, RR Rolf Widmer, dem Zentralsekretär der GDK, Michael Jordi, den Mitgliedern des Projekt-Teams HSM der GDK Katharina Schönbucher und Matthias Fügi (PhD), ferner dem Vorsitzenden der Begleitgruppe Viszeralchirurgie, Prof. Markus Weber, und schliesslich Prof. Markus Zuber sowie Prof. Markus von Flüe (den Viszeralchirurgen im FO) für die kritische Durchsicht des Manuskripts.

\section{Literatur}

1 Birkmeyer JD, Siewers AE, Finlayson EV, Stukel TA, Lucas FL, Batista I, Welch HG, Wennberg DE. Hospital volume and surgical mortality in the United States. N Engl J Med. 2002;346:1128-37.

2 Renzulli P, Lowy A, Maibach R, Egeli RA, Metzger U, Laffer UT. The influence of the surgeon's and the hospital's caseload on survival and local recurrence after colorectal cancer surgery. See comment in PubMed Commons belowSurgery 2006;139:296-304.

3 Güller U, Warschkow R, Ackermann CJ, Schmied B, Cerny T, Ess S. Lower hospital volume is associated with higher mortality after oesophageal, gastric, pancreatic and rectal cancer resection. Swiss Med Wkly. 2017;147:w14473. doi: 10.4414/smw.2017.14473.

4 Archampong D, Borowski DW, Dickinson HO. Impact of surgeon volume on outcomes of rectal cancer surgery: a systematic review and meta-analysis. Surgeon 2010;8:341-52. 